\title{
ENSEÑANZA ORIENTADA A LA ACCIÓN: PROPUESTAS DE LA CÁTEDRA UNESCO DE ARQUITECTURA DE TIERRA, CULTURAS CONSTRUCTIVAS Y DESARROLLO SOSTENIBLE UNITWIN/UPV \\ ACTION-ORIENTED TEACHING: UNESCO SPANISH CHAIR EARTHEN ARCHITECTURE, BUILDING CULTURES AND SUSTAINABLE DEVELOPMENT UNITWIN/UPV
}

\author{
C. Mileto, F. Vegas, V. Cristini, L. García Soriano \\ Universitat Politècnica de València \\ vacri@cpa.upv.es
}

\section{RESUMEN}

En el marco de las propuestas de formación y difusión, promovidas por la Cátedra UNESCO de Arquitectura de Tierra UNITWIN/UPV, vinculada a la Universitat Politècnica de València, destacan los talleres teórico-prácticos constructivos llevados a cabo.

Estas actividades, junto con ciclos de conferencias académicas, constituyen el eje vertebrador de la labor de la Cátedra, centrada en la arquitectura de tierra, las culturas constructivas y el desarrollo sostenible. A continuación se detallan los pormenores de estos talleres, analizando el enfoque metodológico, los objetivos de aprendizaje y los contenidos a desarrollar, así como las características y los detalles de los diferentes módulos didácticos.

Se trata de un abanico variado de actividades, basadas en metodologías activas de aprendizaje, orientadas a labores de análisis y reflexión conjunta, tanto de los docentes como de los alumnos y profesionales, sobre la arquitectura de tierra, su vigencia histórica y su potencial contemporáneo.

Palabras clave: arquitectura de tierra, aprendizaje participativo, metodología activa

\begin{abstract}
Within the framework of UNESCO Spanish Chair "Earthen architecture, building cultures and sustainable development" based in Valencia, at Universitat Politècnica Campus, the authors have carried out a series of educational activities to disseminate a scientific and technical know-how on earthen architecture. The aims of all these actions, thanks to a wide range of communicative and didactical approaches, have been focused on training programs above all for university community, concerning earthen architecture, building cultures and sustainable development. On this basis the text explains details about workshops, methodological approaches, learning aims, contents and didactic modules. A wide range of activities, based on active learning methodologies is presented, with the purpose to underline the features of earthen architecture and its traditional or contemporary potential use.
\end{abstract}

Key words: earthen architecture, active learning, learning by doing 


\section{LA CATEDRA UNESCO DE ARQUITECTURA DE TIERRA, CULTURAS CONSTRUCTIVAS Y DESARROLLO SOSTENIBLE}

La "Cátedra UNESCO de Arquitectura de tierra, culturas constructivas y desarrollo sostenible" se estructura en una red internacional UNITWIN, donde los colaboradores académicos, científicos y profesionales contribuyen a la difusión y la promoción de una ética compartida en lo que se refiere a la formación de profesionales cualificados.

De acuerdo a estos principios, la Cátedra UNESCO Arquitectura de tierra, culturas constructivas y desarrollo sostenible, adscrita a la Escuela Nacional Superior de Arquitectura de Grenoble (ENSAG), integrada al Centro Internacional de la construcción con tierra de dicha institución (CRATerreENSAG), centro de excelencia de la Cátedra, fue creada el 9 de octubre de 1998. Actualmente, la Cátedra cuenta con 41 socios de 26 países diferentes de 4 continentes. La red la componen 10 instituciones en África, 19 instituciones en América, 7 en Asia y 5 instituciones europeas. Los 5 socios europeos son: España (Camilla Mileto y Fernando Vegas, Escuela Técnica Superior de Arquitectura de Valencia), Italia (MaddalenaAchenza, Universidad de Cagliari), Francia (CRATerre-ENSAG), Portugal (Mariana Correia, Escola Superior Gallaecia) y Austria (BASEHabitat).

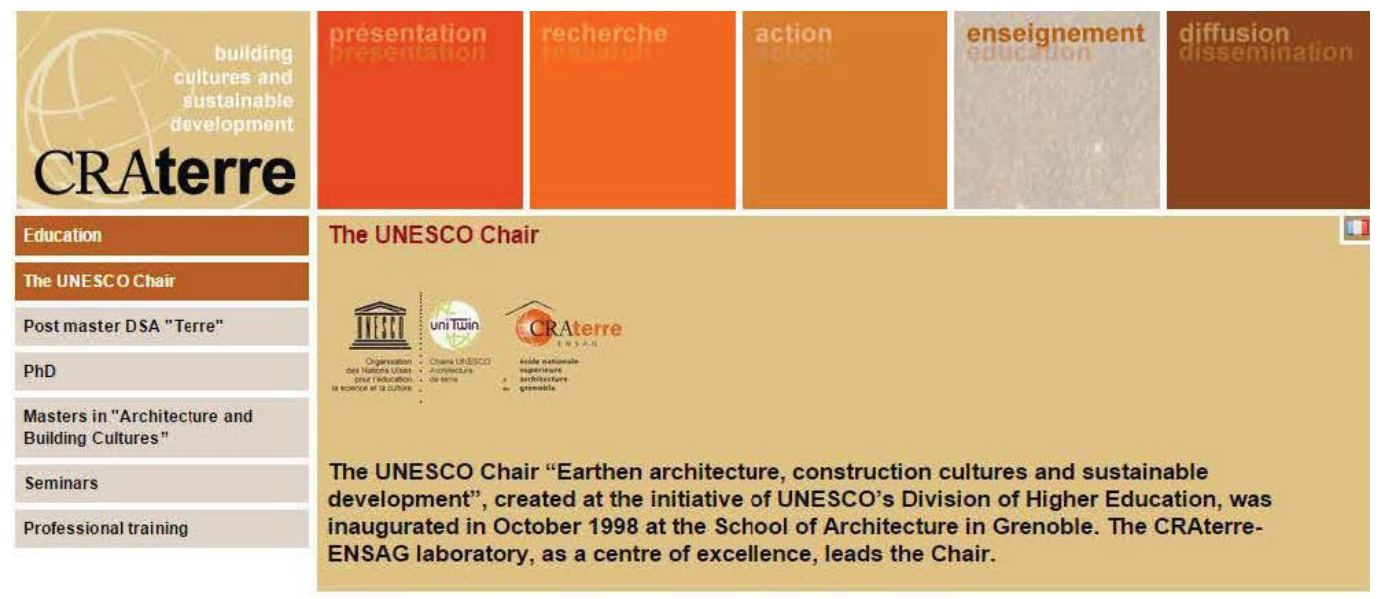

Fig.1. Página web de Cátedra UNESCO Arquitectura de tierra, culturas constructivas y desarrollo sostenible, adscrita a la Escuela Nacional Superior de Arquitectura de Grenoble (ENSAG-http://craterre.org/)

\subsection{La Cátedra Unesco de Arquitectura de Tierra, culturas constructivas y desarrollo sostenible en el Campus UPV de Valencia}

La Cátedra UNESCO de Arquitectura de tierra, culturas constructivas y desarrollo sostenible UNITWIN/UPV es parte de la red de cooperación internacional arriba mencionada y sus objetivos fundamentales son integrar actividades de docencia, investigación y formación; apoyar iniciativas y dinámicas locales, y promover sinergias en el ámbito internacional. De esta manera, la Cátedra favorece y estimula el diálogo entre los diversos países y continentes para facilitar el intercambio y la transferencia del saber hacer.

Esta Cátedra basa su trabajo en la relación entre la educación superior, la formación profesional, la investigación fundamental y aplicada, la información, la documentación y el intercambio en el campo de la arquitectura de tierra. Trata 
tres temas principales: el medioambiente, los asentamientos humanos y el hábitat; la tierra como material y los materiales ecológicos, el medioambiente y el patrimonio.

Los profesores Camilla Mileto y Fernando Vegas, como socios españoles de la cátedra desde 2012, han contribuido en su labor dentro de la Red UNITWIN, promoviendo numerosas actividades de formación y difusión, especialmente entre los propios alumnos de la Universidad.

\section{LOS CONTENIDOS METODOLÓGICOS DESARROLLADOS EN EL MARCO DE LAS ACTIVIDADES PROPUESTAS}

Las propuestas didácticas que se llevan a cabo en el marco de las actividades de la Cátedra Unesco UNITWIN/UPV cuentan principalmente con dos paradigmas pedagógicos, como son la enseñanza orientada a la acción (J. Dewey 1859-1952 y W. Kilpatrick 1871-1965) y la teoría de la actividad (L. Vygotsky 1896-1934 y A. Leóntiev1903-1979). No se trata de esquemas rígidos a aplicar, más bien se trata de enfoques metodológicos muy útiles a la hora de estructurar actividades de aprendizaje participativo, como son algunos de los que la Cátedra Unesco UNITWIN/UPV fomenta en la comunidad universitaria.

\subsection{La enseñanza orientada a la acción y la "teoría de la actividad"}

El primer concepto, basado en la enseñanza orientada a la acción, parte de la voluntad de propiciar una situación concreta que debe-materializarse a través de una actividad práctica. Más adelante, gracias a unas reglas generales extraídas, se logra explicar un principio (procedimiento inductivo). Se trata realmente de un concepto que primero formula el principio, pasando a las leyes que lo rigen o el contexto, y finalmente analiza casos particulares a modo de ejercicio o ejemplo ( J.Bautista Martínez 2012).

Este concepto es esencialmente producto de la didáctica del constructivismo pedagógico que parte del supuesto de que el estudiante se aproxima a la realidad de una forma orientada y selectiva. Con el bagaje acumulado por las distintas actividades, el estudiante, a la luz de lo que está viendo y practicando, formula una serie de hipótesis que sirven como el punto de arranque para iniciar el proceso de elaboración de pautas constructivas definidas (D. Robbis 2003).

El segundo concepto, vinculado a la teoría de la actividad, postula que los estudiantes no son objeto de la instrucción del docente, sino que son aprendices activos, que con oportunos andamiajes son capaces de elaborar para sí y por sí mismos el material que se les provee. Ellos entienden los contenidos sobre la base de sus disposiciones, a partir de los conocimientos previos adquiridos a lo largo de su vida académica (y non).

Para ello la teoría de la actividad se estructura en fases precisas como la búsqueda activa de estrategias de "arranque" de un proceso, la elaboración y ejecución organizada de una actividad práctica, el control durante la realización y la revisión final del proceso.

Por lo tanto, considerando estas dos premisas metodológicas a continuación se tratan las diferentes propuestas académicas llevadas a cabo por la estructura UNITWIN/UPV hasta la fecha. 


\section{ACTIVIDADES DIDACTICAS LLEVADAS A CABO POR LA CÁTEDRA UNESCO UNITWIN/UPV}

La arquitectura de tierra ha estado presente desde épocas muy tempranas en la tradición constructiva de gran parte del territorio de la Península Ibérica, no obstante es, en muchos casos, desconocida o apena valorada por los estudiantes de las escuelas de arquitectura. Por ello, las actividades organizadas en el marco de la cátedra ofrecen a los estudiantes y profesionales un posible acercamiento real a este material, y a las técnicas constructivas vinculadas con la tierra.Por lo tanto, para comprender las finalidades educativas propuestas es importante es destacar dos tipos de actividades: los ciclos de conferencias académicas y los talleres teórico-prácticos académicos.
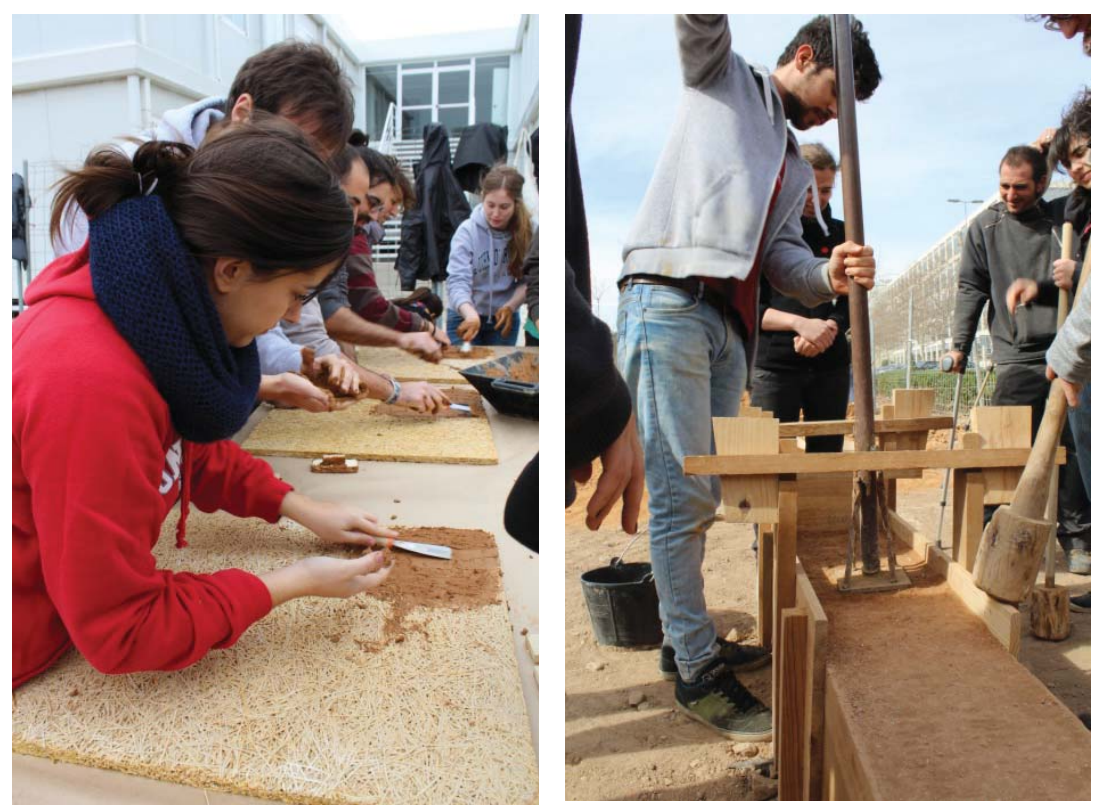

Fig 2-3. Metodología de enseñanza orientada a la acción: ejemplo de actividades llevadas a cabo por Cátedra UNESCO de Arquitectura de Tierra, culturas constructivas y desarrollo sostenible UNITWIN/UPV (authors credits)

\subsection{Ciclos de conferencias académicas}

Una actividad importante desarrollada dentro de la Cátedra es el ciclo de conferencias que se desarrolla en cada curso lectivo en la Escuela de Arquitectura de la UPV, en el que participan tanto alumnos de grado como de postgrado, profesionales y académicos. Se trata de un ciclo en el que las conferencias se organizan de forma periódica, con una conferencia mensual, generalmente el último viernes de cada mes, de modo que los alumnos que participan en el ciclo pueden programar de forma sencilla su asistencia al mismo. Esta actividad mensual pretende acercar a los alumnos a diversas realidades sobre la arquitectura de tierra. Para ello el programa de las ponencias de cada ciclo pretende ser variado en su contenido temático.

Algunos frentes que se han tratado han variado desde temas relacionados directamente con los diversos sistemas constructivos en tierra, tanto en su restauración como en obra nueva hasta contenidos asociados con esta arquitectura como la sostenibilidad (económica, social y cultural) y abarcando 
diversas realidades sociales, geográficas y culturales donde es posible encontrar este tipo de arquitectura (tratando temas geográficamente más lejanos, como Marruecos o Yemen, y otras mucho más cercanos como la barraca valenciana.

Una labor muy importante que se ha podido llevar a cabo, de forma un tanto implícita desde estos ciclos de conferencias, ha sido la elaboración de un listado de alumnos interesados en la arquitectura de tierra que se ha ido incrementando poco a poco y que ha ido configurándose como un grupo de profesionales a los que la cátedra hace difusión de sus actividades para ir creando progresivamente una red de académicos y profesionales vinculados a la investigación y construcción con tierra. Por otro lado, estas conferencias son grabadas y quedan disponibles en la biblioteca de la Escuela de Arquitectura para que los alumnos puedan consultar la información en cualquier momento.

Es importante subrayar, además, que no se trata de clases magistrales "pasivas" que se imparten a una audiencia simplemente "absorbente" (S. Moore \& A. Walsh, Gary y Rísquez 2012). Las conferencias cuentan con momentos de debates, reflexiones, preguntas...aspectos claves a la hora de profundizar un tema o tratar una determinada técnica constructiva. La participación a los eventos es gratuita y este hecho favorece que se produzcan ocasiones de debates con profesionales del sector, expertos externos a la realidad académica...o simplemente interesados al tema, que en muchos casos contribuyen en alimentar enriquecedoras mesas redondas.

\subsection{Talleres académicos}

Como indicado en el punto 2.1 una de las pautas clave de las actividades propuestas por la Cátedra Unesco UNITWIN/UPV está basada en el aprendizaje activo. En este marco, se fomenta especialmente el "Aprender haciendo", conocido también como practica "learning by doing" (metodología de raíz constructivista donde el acentoestá en el aprendizaje directo, evitando las posiciones más radicales de tipo conductivistas, vinculadas a la enseñanza teórica pura- J. Dewey, 1958).

En la práctica, esta metodología puede verse como una inversión del proceso enseñanza-aprendizaje tradicional. En vez de la secuencia habitual que se orienta desde la teoría a la práctica (Teoría $\rightarrow$ Práctica) se invierte el proceso, fomentando una inversión del mismo (Práctica $\rightarrow$ Teoría) (L. Tenenbaum 2011).

Especialmente en la enseñanza de las diversas disciplinas afines al área de arquitectura, es determinante fomentar esta metodología. Realizar una experiencia real, hacer pequeñas prácticas constructivas, vivir la oportunidad de trabajar con un determinado material de construcción (en este caso en particular con la tierra) son algunos de los objetivos que la Cátedra UNESCO UNITWIN/UPV pretende alcanzar. Equivocarse, corregir, hacer de nuevo, mejorar, repetir el ciclo hasta lograr controlar las pautas básicas de las técnicas constructivas a base de tierra son algunos de los modelos didácticos de los talleres propuestos (L. Vigotsky 1896-1934).

Para ello, se han ido ofertando diferentes propuestas, desde talleres básicos sobre las técnicas vinculadas a la tierra como material de construcción (para llevar a cabo principalmente experiencias sobre las propiedades intrínsecas del material tierra y diversas técnicas de construcción como la tapia, el adobe y los 
enlucidos) hasta talleres más específicos, tratando de forma más detallada una técnica constructiva en concreto y analizando temas relativos a mejoras y refuerzos constructivos, donde se afinan y pormenorizan variantes constructivas y estas se aplican posteriormente a proyectos arquitectónicos reales. En estos talleres teórico-prácticos los alumnos, en todo momento, están guiados por expertos y profesionales del sector, a lo largo de diferentes fases de aprendizaje que se pormenorizan a continuación.

Todos los talleres formativos llevados a cabo en el proprio campus de la UPV, cuentan con prácticas constructivas reales, con suministro de materias primas y de herramientas que reflejan la práctica constructiva más autentica, para que la experiencia de los estudiantes pueda ser lo más cercana posible a la realidad profesional.

En este marco los talleres no pretenden alejarse de la teoría constructiva, de hecho las actividades prácticas están siempre complementadas con clases teóricas temáticas, referencias bibliografías y apuntes. No se trata realmente de separar la teoría y la práctica, sino más bien de crear las conexiones necesarias para que dos momentos distintos de aprendizaje se alimenten mutuamente, pero sin ser una matriz rígida de jerarquía académica (K. Bain 2007).
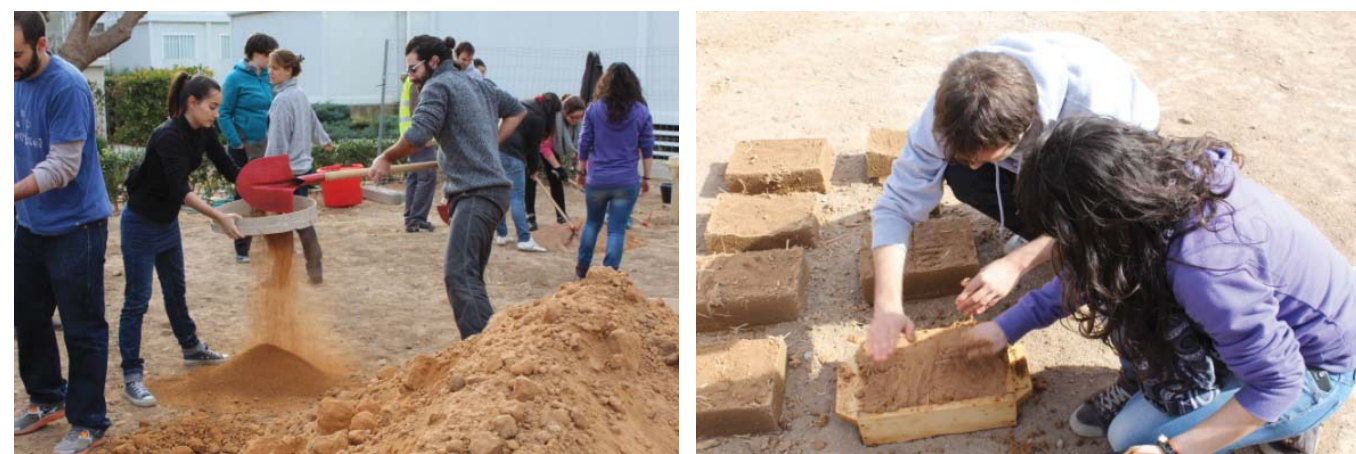

Fig 4-5. Momentos de aprendizaje realizados en algunos de los talleres promovidos por Cátedra UNESCO de Arquitectura de Tierra, culturas constructivas y desarrollo sostenible UNITWIN/UPV (authors credits)

\subsubsection{La organización de los talleres académicos}

Normalmente el modulo de los talleres se articula en 20 horas lectivas de propuestas didácticas variadas, distribuidas durante 2-3 días de clase, estando abiertos a miembros de la comunidad universitaria (estudiantes de grado y postgrado) así como a profesionales. En este marco los talleres cuentan con la siguiente estructura:

1. Clases magistrales/conferencias: docentes o profesionales del sector presentan una serie de casos de estudio, vinculados a proyectos de investigación o trabajos de arquitectura (relativos a proyectos de nueva ejecución, patrimonio histórico, arquitectura vernácula, investigaciones vinculadas a la tierra como material de construcción)

2. Visitas/toma de datos: los docentes proponen actividades vinculadas al estudio empírico de casos reales, bien sean edificios construidos en tierra con un buen estado de conservación, bien sean casos de estudio que presentan 
específicos cuadros patológicos, o proyectos que se encuentren en fase de ejecución/ restauración/ intervención.

3. Propuestas/proyectos: los estudiantes proponen soluciones constructivas vinculadas a distintas técnicas constructivas a base de tierra, detalles y/o mejoras tecnológicas. También se tratan temas vinculados al estudio de patologías, cuadros deformativos o fisurativos de estructuras históricas. Los alumnos promueven pautas constructivas, bien sea de orientación proyectual o más vinculadas a la conservación material de estructuras en tierra.

4. Prácticas de laboratorio/experiencias constructivas: docentes y alumnos colaboran juntos en la realización de auténticos ensayos de laboratorio sobre la tierra como material de construcción (análisis granulométrico del material, pruebas de sedimentación, estudio organoléptico, etc.). Estas prácticas se combinan con la realización de experiencias constructivas reales (elección y dosificación de las materias primas, montaje / desmontaje de soportes, moldes o encofrados, preparación de materiales, puesta en obra, etc.).

Los talleres no cuentan con un esquema rígido, estos cuatro bloques de aprendizaje pueden tener un protagonismo distinto dentro de cada taller temático, cubriendo un número superior o inferior de horas según las propuestas y los objetivos didácticos a alcanzar. Sin duda cada área didáctica es crucial para adquirir habilidades y destrezas constructivas vinculadas a la tierra como material de construcción, y cada una de ella se fomenta con proporción variable.

Más en detalle, durante el curso 2013-2014 se han promovido tres talleres teórico-prácticos, que se han distribuido temporalmente durante el curso lectivo, los meses de febrero, mayo y julio, ofreciendo a los alumnos una formación continuada, optativa y complementaria a sus actividades obligatorias en la escuela.

Estos talleres han tenido una gran acogida por la comunidad universitaria y por los diversos profesionales del sector, reuniendo en cada una de las actividades más de 50 participantes, por lo que han sido gratas experiencias de encuentro, discusión y aprendizaje, y sin duda estas actividades van a seguir realizándose y ampliándose en los sucesivos cursos lectivos.

\section{CONCLUSIONES}

Considerando las bases metodológicas de la enseñanza orientada a la acción y la "teoría de la actividad", el texto presentado subraya la reciente trayectoria de la Cátedra Unesco UNITWIN/UPV, tras sus primeros años de recorrido, mostrando las distintas áreas didácticas y propuestas formativas que se han llevado a cabo. Se trata de un abanico variado de actividades que fundamentalmente aspirana fomentar en los alumnos hábitos ambientales, culturales y sociales volcados en el desarrollo sostenible, empleando el "material tierra", por definición natural, no contamínante, económico...como herramienta clave de formación y difusión de estos principios.

La organización de ciclos de conferencias y talleres académicos insisten en estructurar un aprendizaje activo sobre los temas indicados, a través de un proceso de aprendizaje orientado, participativo y práctico. Además, hasta la fecha, los alumnos han mostrado un nivel de aceptación, entusiasmo y atención 
creciente hacia los temas tratados. La buena acogida de las actividades propuestas es sin duda un feed back interesante a considerar y motivador para seguir desarrollando propuestas didácticas y mejorarlas. Se trata además de dinámicas que los nuevos planes de estudio siguen propiciando y respaldando. Este hecho hace que las actividades promovidas por Cátedra Unesco UNITWIN/UPV tengan un potencial creciente, cuya trayectoria sigue abierta y viva hacia futuros escenarios didácticos y posibles mejoras académicas.

\section{BIBLIOGRAFÍA}

http://unesdoc.unesco.org/images/0014/001439/143918F.pdf

http://craterre.org/enseignement:chaire-unesco/?new lang=en GB

Bautista Martínez, J. (2012), Innovación en la universidad: prácticas, políticas y retóricas, Grao Ed., Barcelona

Bain, K.,(2007) Lo que hacen los mejores profesores universitarios, UV Ed., Valencia

Dewey, J. (1958). Experiencia y Educación, Biblioteca Nueva Ed., Madrid, $11^{\mathrm{a} e d .}$ Ferrándiz García, C. (2005), Evaluación y desarrollo de la competencia cognitiva, un estudio desde el modelo de las competencias múltiples, Ministerio de educación y Ciencia Ed., Madrid

Morin, E. (1999), Los siete saberes que necesita la educación del futuro, UNESCO Ed., Paris

Moore, S. \& Walsh, Gary y Rísquez, A. (2012), Estrategias Eficaces Para Enseñar En La Universidad. Guía Para Docentes Comprometidos, Narcea Ed., Madrid

Robbis, D. (2003), Vygotsky's and A.A. Leontiev's Semiotics and Psycholinguistics: Applications for Education, Second Language Acquisition, and Theories of Language, Praeger Publisher, EE.UU

Tenenbaum, S. (2011), William Heard Kilpatrick: Trail Blazer in Education, Literary Licensing Ed, NY

Vigotsky, L. (2004), Teoría de las emociones, estudio histórico-psicologíco, Akal Ed., Madrid 